\title{
A text mining framework for accelerating the semantic curation of literature
}

DOI:

10.1007/978-3-319-43997-6_44

\section{Document Version}

Accepted author manuscript

Link to publication record in Manchester Research Explorer

\section{Citation for published version (APA):}

Batista-Navarro, R., Hammock, J., Ulate, W., \& Ananiadou, S. (2016). A text mining framework for accelerating the semantic curation of literature. In Research and Advanced Technology for Digital Libraries : 20th International Conference on Theory and Practice of Digital Libraries, TPDL 2016 Hannover, Germany, September 5-9, 2016 proceedings (pp. 459-462). (Lecture Notes in Computer Science (including subseries Lecture Notes in Artificial Intelligence and Lecture Notes in Bioinformatics); Vol. 9819). Springer Nature. https://doi.org/10.1007/978-3-31943997-6_44

\section{Published in:}

Research and Advanced Technology for Digital Libraries :

\section{Citing this paper}

Please note that where the full-text provided on Manchester Research Explorer is the Author Accepted Manuscript or Proof version this may differ from the final Published version. If citing, it is advised that you check and use the publisher's definitive version.

\section{General rights}

Copyright and moral rights for the publications made accessible in the Research Explorer are retained by the authors and/or other copyright owners and it is a condition of accessing publications that users recognise and abide by the legal requirements associated with these rights.

\section{Takedown policy}

If you believe that this document breaches copyright please refer to the University of Manchester's Takedown Procedures [http://man.ac.uk/04Y6Bo] or contact uml.scholarlycommunications@manchester.ac.uk providing relevant details, so we can investigate your claim.

\section{OPEN ACCESS}




\title{
A Text Mining Framework for Accelerating the Semantic Curation of Literature
}

\author{
Riza Batista-Navarro ${ }^{1}$, Jennifer Hammock ${ }^{2}$, and William Ulate $^{3}$ \\ and Sophia Ananiadou ${ }^{1}$ \\ 1 School of Computer Science, University of Manchester, M13 9PL, United Kingdom \\ riza.batista@manchester.ac.uk \\ 2 Smithsonian Institute, Washington, D.C., USA \\ 3 Missouri Botanical Garden, Missouri, USA
}

\begin{abstract}
The Biodiversity Heritage Library is the world's largest digital library of biodiversity literature. Currently containing almost 40 million pages, the library can be explored with a search interface employing keyword-matching, which unfortunately fails to address issues brought about by ambiguity. Helping alleviate these issues are tools that automatically attach semantic metadata to documents, e.g., biodiversity concept recognisers. However, gold standard, semantically annotated textual corpora are critical for the development of these advanced tools. In the biodiversity domain, such corpora are almost non-existent especially since the construction of semantically annotated resources is typically a timeconsuming and laborious process. Aiming to accelerate the development of a corpus of biodiversity documents, we propose a text mining framework that hastens curation through an iterative feedback-loop process of (1) manual annotation, and (2) training and application of statistical concept recognition models. Even after only a few iterations, our curators were observed to have spent less time and effort on annotation.
\end{abstract}

\section{Introduction}

The Biodiversity Heritage Library (BHL) holds the largest collection of legacy literature on biodiversity, contributed by a number of botanical and natural history libraries striving to make their content available to the public through digitisation efforts (e.g., scanning, optical character recognition, bibliographic indexing). As a result, more than 100,000 titles are now publicly accessible through the digital library, equivalent to around 40 million pages of taxonomic literature.

To allow its users to find information of interest, BHL provides a search system that facilitates keyword-based matching of user-supplied queries against bibliographic metadata, e.g., book titles. It is not rare, however, for keywords to have ambiguous senses. Methods which are more sophisticated than keywordbased matching are thus necessary for ensuring that the results returned by BHL are relevant to a user's query. For example, to help distinguish between a word's several senses, one could incorporate a tool that will automatically mark up mentions of concepts within text and assign them into predefined categories: an 
information extraction task known as named entity recognition (NER). Amongst the many NERs which have emerged, machine learning-based ones have demonstrated superior performance owing to their stronger ability to generalise on unseen cases, based on samples presented to them during model training. The drawback, however, is their reliance on the availability of textual corpora with gold standard semantic annotations. Whilst such corpora proliferate in a few domains, e.g., newswire, biomedicine, some subject areas such as biodiversity are relatively low-resourced. To the best of our knowledge, there has only been one corpus of biodiversity documents which was semantically enriched through manual annotation of taxonomic names ${ }^{1}$. This scarcity in resources can be attributed to the costly time and human effort required by corpus construction, especially in cases where the target domains are specialised.

In order to support the development of advanced NER techniques for the purpose of automatically categorising concepts in BHL documents, we pursued the construction of a gold standard annotated corpus of biodiversity literature. We however innovate the annotation process to reduce the time and effort required from curators. Specifically, we propose a text mining-based framework driven by an iterative feedback-loop mechanism that (1) gathers manual annotations from curators, (2) employs the conditional random fields (CRF) algorithm [1] to train NER models on manual annotations gathered so far, and (3) applies the trained models on unannotated documents which will then be manually validated by curators. The framework is implemented as processing workflows in $\mathrm{Argo}^{2}$, a Web-based text mining workbench [2]. Following the Unstructured Information Management Architecture (UIMA) standard, Argo integrates various natural language processing (NLP) tools into workflows. Furthermore, it supports the execution of semi-automatic workflows which allow curators to manually provide or revise annotations through a user-interactive annotation editor.

\section{Methods}

As a preliminary step, we asked our partners from the Missouri Botanical Garden, one of the botanical libraries behind BHL, to select 10 titles in the BHL collection to form the basis of the corpus. Based on this process, a total of 145 pages were collected, comprising our new corpus which was annotated by two curators according to the approach outlined below.

\subsection{Preparation of the annotation schema}

With the guidance of our partner biodiversity specialist, we identified the semantic categories which define concept types that should be annotated by the curators. The following seven categories were selected: (1) taxonomic entity, (2) geographic location, (3) habitat, (4) temporal expression, (5) person, (6) anatomical entity, and (7) quality. A detailed set of annotation guidelines was prepared

\footnotetext{
${ }^{1}$ https://github.com/VBRANT/vibrantcorpus

${ }^{2}$ http://argo.nactem.ac.uk
} 
to elucidate cases that should be included and how they should be demarcated ${ }^{3}$. Since Argo is a generic text mining workbench and does not cater to any specific application or domain, we configured its annotation editor by means of a new UIMA type system formally representing our semantic categories of interest.

\subsection{Selection of controlled vocabularies}

The NER task was cast as a sequence labelling problem, making use of sentence tokens as the fundamental units of analysis. Features representing tokens are therefore key in the training and subsequent application of conditional random field models for NER. Our previous work on developing CRF-based NERs showed that a boost in performance can be obtained through the incorporation of semantic features, e.g., matches between tokens in text and entries in controlled vocabularies [3]. We generated our semantic features based on the Catalogue of Life and the following ontologies: the Ontology of Biological Attributes, and the Gazetteer, Environment, Uber Anatomy, Phenotype and Trait, and Flora Phenotype Ontologies. The names and synonyms contained in these resources were compiled using the NERsuite package ${ }^{4}$, our chosen implementation of CRFs. The manner in which our framework utilised the compiled dictionaries is described in the next section.

\subsection{Machine learning-based training and tagging workflows}

The framework is initiated by curators upon their creation of new annotations for a small number of documents, e.g., two pages, using Argo's annotation editor. These seed annotations are saved in XML Metadata Interchange (XMI) files, UIMA's native serialisation format. Our feedback-loop mechanism is realised as the iterative execution of two workflows: (1) a completely automatic workflow for training CRF models, and (2) a semi-automatic one for applying the newly trained models on unseen documents and presenting their results to human curators for revisions. The first workflow (Figure 1a), begins with an XMI Reader which loads documents from a directory containing manually provided annotations (i.e., training documents). After the Reader is a series of NLP components for pre-processing, i.e., sentence splitting, tokenisation, lemmatisation and part-of-speech tagging. These components are succeeded by instances of the NERsuite Dictionary Feature Generator, which load pre-compiled dictionaries (described in the previous section) to generate semantic features based on token matches. Next in the workflow are instances of the NERsuite Trainer component, each of which generates character and word $n$-grams according to the outputs of pre-processing tools. Together with the previously captured dictionary matches, these features are learned by the NER models during training.

The second workflow (Figure 1b) similarly begins with an XMI Reader, albeit one for loading unannotated documents. Similar components for pre-processing

\footnotetext{
${ }^{3}$ http://wiki.miningbiodiversity.org/doku.php?id=guidelines

${ }^{4}$ http://nersuite.nlplab.org
} 


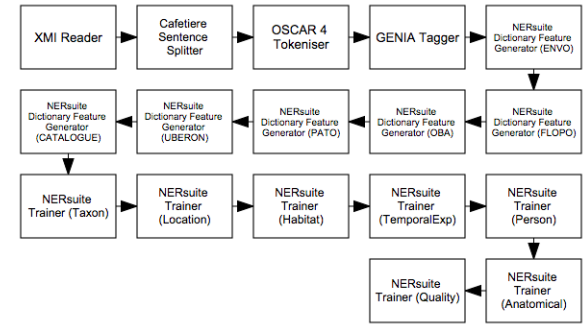

(a) Training of NER models

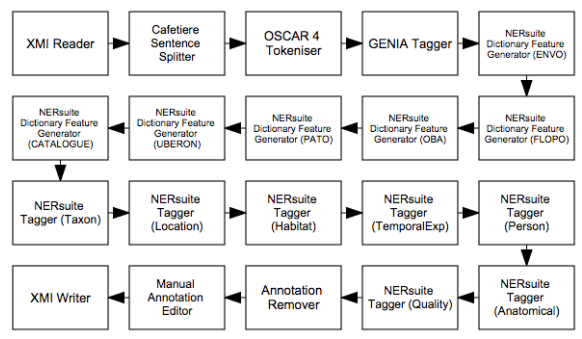

(b) Application of NER models and manual revision

Fig. 1: Workflows for accelerating biodiversity concept curation

and semantic feature generation follow. However, these are instead succeeded by NERsuite Tagger components which apply the previously trained models to annotate semantic concepts in the documents of the current set. These automatically annotated documents are then presented by the editor to the curators for revision. The revised annotations are saved as XMI files in the curators' directory of training documents, thus incrementally increasing the amount of gold standard annotations used for model retraining in succeeding iterations.

\section{Discussion and Conclusion}

As the framework progresses with the iterative execution of the workflows described above, the amount of annotated data increases, allowing the models to become better at learning the NER task. In turn, the quality of annotations generated by the models on unseen documents also improves. There is therefore a progressive decrease in the amount of time and effort required from the curators in revising automatically generated annotations. Our two curators empirically confirmed this, observing that whilst in the first two to four iterations they had to revise a huge proportion of the automatically generated annotations, by the seventh or eighth iteration they were supplying substantially less corrections.

\section{References}

1. Lafferty, J.D., McCallum, A., Pereira, F.C.N.: Conditional random fields: Probabilistic models for segmenting and labeling sequence data. In: Proceedings of the Eighteenth International Conference on Machine Learning. pp. 282-289. ICML '01, Morgan Kaufmann Publishers Inc., San Francisco, CA, USA (2001)

2. Rak, R., Rowley, A., Black, W., Ananiadou, S.: Argo: an integrative, interactive, text mining-based workbench supporting curation. Database: the journal of biological databases and curation 2012, bas010 (2012)

3. Batista-Navarro, R., Rak, R., Ananiadou, S.: Optimising chemical named entity recognition with pre-processing analytics, knowledge-rich features and heuristics. Journal of Cheminformatics 7(Suppl 1), S6 (2015) 